\title{
Flavoring agents present in a dentifrice can modify volatile sulphur compounds (VSCs) formation in morning bad breath
}

Daiane Cristina Peruzzo(a) Sérgio Luis Salvador ${ }^{(b)}$ Antonio Wilson Sallum(c) Getúlio da Rocha Nogueira-Filho(c)

(a) PhD Student; (c) Professors - Department of Prosthodontics and Periodontics, Piracicaba Dental School, State University of Campinas (FOP - Unicamp), Piracicaba, SP, Brazil.

(b) Professor, Department of Clinical Analysis, School of Pharmaceutical Sciences of Ribeirão Preto, University of São Paulo (USP), Ribeirão Preto, SP, Brazil.

\section{Corresponding author:}

Daiane Cristina Peruzzo

Av. Evandro Batista Vieira, 775/13

Alphaville - Campinas - SP

CEP: 13098-390

E-mail:daiaperuzzo@yahoo.com.br

Received for publication on Feb 27, 2007

Accepted for publication on Sep 03, 2007

\begin{abstract}
This study aimed to evaluate the effects of a flavor-containing dentifrice on the formation of volatile sulphur compounds (VSCs) in morning bad breath. A two-step, blinded, crossover, randomized study was carried out in 50 dental students with a healthy periodontium divided into two experimental groups: flavor-containing dentifrice (test) and non-flavor-containing dentifrice (control). The volunteers received the designated dentifrice and a new toothbrush for a $3 \mathrm{X} /$ day brushing regimen for 2 periods of 30 days. A seven-day washout interval was used between the periods. The assessed parameters were: plaque index (PI), gingival index (GI), organoleptic breath scores (ORG), VSC levels (as measured by a portable sulphide monitor) before (H1) and after (H2) cleaning of the tongue, tongue coating (TC) wet weight and BANA test from TC samples. The intra-group analysis showed a decrease in ORG, from 3 to 2, after 30 days for the test group $(\mathrm{p}<0.05)$. The inter-group analysis showed lower values in ORG, $\mathrm{H} 1$ and $\mathrm{H} 2$ for the test group $(\mathrm{p}<0.05)$. There was no difference between the amount of TC between groups and the presence of flavor also did not interfere in the BANA results between groups $(\mathrm{p}>0.05)$. These findings suggest that a flavorcontaining dentifrice seems to prevent VSCs formation in morning bad breath regardless of the amount of TC in periodontally healthy subjects.
\end{abstract}

Descriptors: Halitosis; Volatile sulfur compounds; Dentifrices; Flavoring agents; BANA test. 


\section{Introduction}

Bad breath or halitosis is caused by the presence of volatile sulphur compounds (VSCs) in the exhaled air, particularly methyl mercaptan $\left(\mathrm{CH}_{3} \mathrm{SH}\right)$ and hydrogen sulphide $\left(\mathrm{H}_{2} \mathrm{~S}\right)^{1}$, in addition to shortchain organic fatty acids such as propionic acid, butyric acid, valeric acid and polyamines, putrescine, cadaverine and skatoles. ${ }^{2}$ In vitro studies have demonstrated that gram-negative anaerobic bacteria are capable of producing volatile sulfur compounds from protein products. ${ }^{3}$

During sleep, the proliferation of oral microorganisms associated with nocturnal hypo-salivation is responsible for the greater production of VSCs that promotes morning bad breath, even in periodontally healthy subjects (PHS). ${ }^{4}$ Strategies for controlling bad breath are related to the control of bacteria growth, especially proteolytic bacteria, and involve the debridement of teeth and the tongue in combination with the use of antibacterial agents. ${ }^{5,6}$ For this purpose, a great variety of products have been used to inhibit or mask bad breath, including gums, oral rinses and dentifrices. Studies have shown the effectiveness of toothpastes containing different antimicrobial agents for bad-breath control: Sharma et al. ${ }^{4}$ (1999) and Nogueira-Filho et al. ${ }^{7}$ (2002) demonstrated that dentifrices containing triclosan were able to reduce oral malodor. In the same way, studies by Niles et al. ${ }^{8}$ (1993) and Brunette et al. ${ }^{9}$ (1998) showed the efficacy of sodium bicarbonate in reducing the levels of VSCs, while Gerlach et al. ${ }^{10}$ (1998) showed that toothpastes containing stannous fluoride are also effective in reducing oral malodor. In addition, the findings of the study performed by Peruzzo et al. * suggest that sodium lauryl sulphate, present in dentifrices, appears to prevent VSCs formation in morning bad breath in PHS. Some authors ${ }^{11,12}$ have suggested that the presence of flavoring agents could stimulate saliva flow, modifying the clearance of the oral cavity, speeding up the elimination of bacteria by swallowing and altering the development of oral malodor. However, no study has evaluated the possible effects of flavor- ing agents, present in dentifrices, on oral malodor. Thus, the main objective of this study was to evaluate the effect of the flavoring agents present in a dentifrice on the formation of VSCs in the morning breath of healthy individuals.

\section{Material and Methods Subjects}

Fifty volunteers were selected among students from the School of Dentistry of Piracicaba, State University of Campinas (UNICAMP, SP, Brazil). Twenty-seven men and 23 women, between the ages of 18 and 30 years (mean age $24 \pm 4.6$ years), who agreed to participate in the study and signed an informed consent, and who presented no systemic disorders were included in this study. The following subjects were excluded: smokers, pregnant women, individuals who had taken antibiotics over the last 6 months or permanently used any drugs and/or presented otorhinolaryngological and/or respiratory problems. These excluding conditions were investigated in a detailed anamnesis. With regard to clinical criteria, individuals wearing appliances or orthodontic contention devices, and presenting deep tongue fissures, dental implants, prostheses or badly adapted restorations were also excluded from the study. In addition, the participants in the study should present a normal salivary flow rate $(1.5-2.5 \mathrm{ml})$ and at least 24 teeth that did not present gingival probing depths $(\mathrm{PD})>3 \mathrm{~mm}$ and gingival indexes (GI) and plaque indexes $(\mathrm{PI})=1$ in more than $10 \%$ of sites.

\section{Ethical aspects}

The present study had previously obtained approval by the FOP-UNICAMP Human Research Ethics Committee (Protocol \# 062/2003). The volunteers signed an informed consent, according to the Helsinki II Declaration and the Dentistry Ethical Code (CONEP/MS, Brazil).

\section{Test and control products}

Two dentifrices were compared: a flavor-containing test dentifrice (Close Up ${ }^{\circledR}$ Red - Unilever,

\footnotetext{
*In press. Peruzzo DC, Salvador SL, Sallum AW, Nogueira-Filho GR. Effects of sodium lauryl sulphate, present in dentifrice, on volatile sulphur compound formation in morning bad breath. J Int Acad Periodontol. (Accepted for publication in April 2007).
} 
Vinhedo, SP, Brazil) and a non-flavor-containing dentifrice (control) that presented the same formulation as the test dentifrice, except for the flavor. Both dentifrices were produced and supplied by Unilever (Vinhedo, SP, Brazil) and were placed, by the maker, in white new plastic coded tubes in such a way that direct identification of the product was impossible. The codes were not disclosed until the study had been completed.

\section{Study design}

A crossover, blind study was carried out on a sample of 50 healthy individuals, randomly (by a tossed coin) assigned to two experimental groups: test and control. The volunteers received the designated dentifrice and a new toothbrush for a $3 x /$ day-brushing regimen for 2 periods of 30 days each. A washout interval of 7 days occurred between the periods, in which all the volunteers used the control dentifrice to avoid a possible carry-over effect. Assessments were performed by one single calibrated examiner.

\section{Clinical assessment and pre-experimental phase}

Seven days prior to the first experimental period, the following clinical parameters were evaluated: PI (Plaque Index), ${ }^{13}$ GI (Gingival Index), ${ }^{13}$ PD (Probing Depth), gingival recession (GR) and clinical attachment level (CAL). These measurements were performed to ensure that the subjects fitted the selection criteria, and did not have any type of periodontal disease. Professional removal of calculus using Grace curetes (Neumar, São Paulo, SP, Brazil) was performed as well as dental biofilm removal with rubber cups and prophylactic paste. All volunteers received the control dentifrice and a new toothbrush (Close Up ${ }^{\circledR}$, Unilever, Vinhedo, SP, Brazil) and written instructions to use the dentifrice three times a day for 7 days. They were also asked to suspend the use of any type of tongue cleaning or mouth rinses. The use of dental floss was not restricted, considering the crossover design of the study, in which individual variables could be compensated. These measures were performed such as to guarantee that the volunteers started with the same gingival conditions.

\section{Experimental phase}

After the pre-experimental phase, the volunteers were scheduled for an appointment at 7 a.m. for breath analysis, in compliance with the following criteria: the night before the appointment, volunteers were required not to ingest spicy foods, with garlic or onions, or alcoholic beverages, and the last tooth brushing had to be done before 12 p.m. In the morning, volunteers should be in absolute fasting, without performing any type of oral hygiene and should not use any cosmetics that release odors/perfumes. ${ }^{6}$

The following parameters were assessed: an organoleptic breath measurement (ORG), VSCs levels as measured by a portable sulphide monitor before (H1) and after cleaning of the tongue $(\mathrm{H} 2)$, tongue coating (TC) wet weight, PI, GI and BANA test from TC samples on days 0 and 30 of each experimental period. According to the crossover design, the volunteers received one of the assigned dentifrices and a new toothbrush (Close Up ${ }^{\circledR}$, Unilever, Vinhedo, SP, Brazil) that were used during the following 30 -day periods.

\section{Organoleptic analyses}

Individuals were asked to keep their mouths completely closed for 3 minutes, breathing only through the nose. After this time had elapsed, the volunteer was instructed to release the air slowly by mouth, $10 \mathrm{~cm}$ distant from the examiner's nose, ${ }^{14}$ who was previously calibrated for this purpose $(k$ index $=0.89$ ) and blind to which group each individual belonged to. A score from 0 to 5 was then attributed according to Rosenberg's ${ }^{15}$ scale.

\section{VSCs measurements}

Quantitative measurements of VSCs were performed with the use of a portable industrial sulphide monitor (Halimeter ${ }^{\circledR}$ RH-17E, Interscan Corp., Chatsworth, CA, USA). Each patient's maximum peak of VSCs was determined in ppb through direct reading on the monitor analogical scale. This procedure was carried out in each volunteer at all times before and after TC removal.

\section{Tongue coating removal and collection}

The TC was removed by the use of sterile tongue scrapers (Saudbucal ${ }^{\circledR}$, São Paulo, SP, Brazil) on the 


\begin{tabular}{|c|c|c|c|c|c|}
\hline & & & & & \\
\hline Table 1 - Comparisons & & Contro & Group & Test & Sroup \\
\hline 30 for the test and control & Measurements & Day 0 & Day 30 & Day 0 & Day 30 \\
\hline groups (mean $\pm \mathrm{SD}$; & PI (\% mean) & $1.19 \pm 1.75^{\mathrm{Aa}}$ & $1.40 \pm 1.16^{\mathrm{A}}$ & $1.12 \pm 1.61^{\mathrm{Aa}}$ & $1.09 \pm 1.48^{\mathrm{A}}$ \\
\hline & Gl (\% mean) & $2.14 \pm 2.35^{\mathrm{Aa}}$ & $2.25 \pm 2.11^{\mathrm{A}}$ & $2.31 \pm 2.57^{\mathrm{Aa}}$ & $2.18 \pm 2.29^{A}$ \\
\hline & ORG (score medians) & $3^{\mathrm{Aa}}$ & $3^{\mathrm{A}}$ & $3^{A a}$ & $2^{\mathrm{B}}$ \\
\hline & $\mathrm{Hl}$ (ppb) & $171.7 \pm 162.4^{\mathrm{Aa}}$ & $173.92 \pm 98.1^{\mathrm{A}}$ & $129.2 \pm 73.4^{\mathrm{Aa}}$ & $132.6 \pm 89.7^{\mathrm{A}}$ \\
\hline & $\mathrm{H} 2$ (ppb) & $169.7 \pm 145.2^{\mathrm{Aa}}$ & $197.5 \pm 130.5^{\mathrm{A}}$ & $130.9 \pm 73.4^{\mathrm{Aa}}$ & $141.9 \pm 131^{\mathrm{A}}$ \\
\hline & $\mathrm{TC}(\mathrm{g})$ & $0.18 \pm 0.10^{\mathrm{Aa}}$ & $0.21 \pm 0.11^{\mathrm{A}}$ & $0.2 \pm 0.17^{\mathrm{Aa}}$ & $0.17 \pm 0.11^{\mathrm{A}}$ \\
\hline & BANA(+) & $27^{A a}$ & $23^{A}$ & $28^{\mathrm{Aa}}$ & $22^{\mathrm{A}}$ \\
\hline
\end{tabular}

Means followed by distinct capital letters in lines differ statistically (intra-group, $p<0.05$ ). Means followed by distinct lower case letters in lines differ statistically for day 0 (inter-group, $p<0.05$ ).

posterior third of the patient's tongue. Five backto-front movements were performed to remove the coating, which was deposited in a pre-weighed sterile recipient. The recipient containing the TC was weighed again and a wet weight value was recorded.

\section{Enzymatic assay - BANA test}

According to Loesche et al. ${ }^{16}$ (1990) and Loesche, Kazor $^{17}$ (2002) the BANA reagent card (Knowell, Therapeutic Technologies Inc., Toronto, ON, Can$\mathrm{ada}$ ) is used on a portion of the previously weighed TC samples that were placed in an incubator at $55^{\circ} \mathrm{C}$ for 15 minutes. Results were scored as either blue spots (positive) or no color change (negative).

\section{Statistical analysis}

The Kolmogorov-Smirnov test was used to verify the normality of the data and two non-parametric tests were used. After calculating all the means for days 0 and 30 for each experiment, the Wilcoxon non-parametric test was applied to the data. For all of the analysis, a 5\% significance level was established and data were analyzed using the BioEstat 3.0 software. ${ }^{18}$

\section{Results}

All selected volunteers, 27 men and 23 women, between the ages of 18 and 30 years, completed this study. None of the volunteers complained about bad breath during the day and all participants reported having regular yearly dental examinations and
Table 2 - Inter-group comparisons at day 30 (mean \pm SD; $\mathrm{n}=50)$.

\begin{tabular}{l|c|c}
\hline \multicolumn{1}{c|}{ Measurements } & Control & Test \\
\hline $\mathrm{Pl}(\%$ mean) & $1.40 \pm 1.16^{\mathrm{A}}$ & $1.09 \pm 1.48^{\mathrm{A}}$ \\
\hline $\mathrm{Gl}(\%$ mean) & $2.25 \pm 2.11^{\mathrm{A}}$ & $2.18 \pm 2.29^{\mathrm{A}}$ \\
\hline ORG (score medians) & $3^{\mathrm{A}}$ & $2^{\mathrm{B}}$ \\
\hline $\mathrm{H} 1(\mathrm{ppb})$ & $173.92 \pm 98.1^{\mathrm{Ab}}$ & $132.6 \pm 89.7^{\mathrm{Ba}}$ \\
\hline $\mathrm{H} 2(\mathrm{ppb})$ & $197.5 \pm 130.5^{\mathrm{Aa}}$ & $141.9 \pm 131^{\mathrm{Ba}}$ \\
\hline TC $(\mathrm{g})$ & $0.21 \pm 0.11^{\mathrm{A}}$ & $0.17 \pm 0.11^{\mathrm{A}}$ \\
\hline BANA(+) & $23^{\mathrm{A}}$ & $22^{\mathrm{A}}$ \\
\hline
\end{tabular}

Means followed by distinct capital letters in lines differ statistically $(p<0.05)$. Means followed by distinct lower case letters in columns differ statistically $(\mathrm{H} 1$ and $\mathrm{H} 2 ; \mathrm{p}<0.05)$.

brushing their teeth at least three times a day.

The intra-group comparisons, between day 0 and day 30 for groups, and the inter-group comparisons for day 0 between groups are shown in Table 1.

Table 2 shows the inter-group comparisons at day 30 for the control and test groups and the intragroup comparisons for $\mathrm{H} 1 / \mathrm{H} 2$. The comparisons between treatments, after 30 days, demonstrated lower values for ORG, $\mathrm{H} 1$ and $\mathrm{H} 2$ for the test group $(\mathrm{p}<0.05)$. There was no difference in PI, GI, nor in the amount of TC between test and control groups. TC removal resulted in an increase in the VSCs level from $\mathrm{H} 1$ to $\mathrm{H} 2$ only for the control group. When the groups were statistically compared among periods, the presence of flavor did not interfere in the BANA results $(\mathrm{p}>0.05)$. 


\section{Discussion}

In order to reduce morning bad breath, two approaches have been used and tested in the literature: cleaning the tongue and tooth surfaces by physical methods ${ }^{5,19}$ and/or reducing the bacterial loads by chemical agents present in dentifrices and mouthrinses. ${ }^{7,11,17}$ In the present study, the presence of flavoring agents in a commercial dentifrice seemed to reduce morning bad breath in healthy subjects by decreasing the formation of VSCs levels; this effect was not related to the amount of tongue coating nor to PI and GI, which did not differ between the groups.

A number of reports ${ }^{5,19-23}$ show that the amount of TC is closely correlated to malodor. Miyazaki et al. ${ }^{22}$ (1995) evaluated oral malodor using a portable sulphide monitor in 2,672 individuals and observed a high correlation between VSCs and TC in all age groups. De Boever, Loesche ${ }^{21}$ (1995) examined 16 people who complained of oral malodor and demonstrated that mouth odor was significantly related both to tongue odor and to the amount of TC, which was estimated visually as absent, light, moderate or heavy. In the present study there was a reduction in the scores of ORG and in the VSCs, measured by a monitor (H1 and $\mathrm{H} 2$ ), in the test group, without significant changes in the amount of TC. In contrast with our study, these studies ${ }^{21,22}$ examined both periodontally-compromised and healthy subjects, which may have contributed to increase the association between tongue coating and malodor parameters. In addition, Yaegaki, Sanada ${ }^{23}$ (1992) found that removal of TC markedly reduced VSCs production, as measured by gas chromatography. However, the present results demonstrated that VSCs levels, measured after TC removal (H2), increased in the control group, which could be explained by the volatilization of gases, as an imediate result of TC removal. This increase was not found in the test group, which could indicate that the presence of flavor might be interfering in VSCs volatilization.

Organoleptic scores, or the use of one's nose to smell and rank the intensity of the odors emanating from a mouth, is the gold standard for the measurement of oral malodor, as it reflects the realtime presence of an objectionable odor, as detected by a judge. ${ }^{4}$ According to some studies ${ }^{5,15}$ there is a significant correlation between the odor scores measured by judges and the VSCs levels recorded by a sulphide monitor. Goldberg et al. ${ }^{2}$ (1994) also reported that, in addition to the VSCs measured by a sulphide monitor, there are other foul smelling elements that are not captured by the monitor, and may be perceived by the human olfactory sense. In this report, a trained, blind and calibrated judge detected differences for the ORG scores between groups.

Loesche, Kazor $^{17}$ (2002) reported that most cases of oral malodor are the result of the proteolytic activity of bacteria, and that three species (Treponema denticola, Porphyromonas gingivalis and Tanerella forsythia) could be responsible for this activity. ${ }^{3}$ These microorganisms can be detected in TC samples using the BANA test. ${ }^{16}$ Studies with individuals with halitosis demonstrated that the TC samples were positive for the BANA test ${ }^{20,21}$ and that the TC of individuals with high ORG scores were related to a greater positive BANA result. In the present study, there were no differences in the number of positive results in the BANA test between periods, indicating an absence of high loads of proteolytic microorganisms in the TC. In addition, the subjects of the present study were periodontally healthy and did not complain about halitosis. The results of the present investigation indicated a decrease in VSCs levels and ORG scores with the use of a flavor-containing dentifrice, which is in agreement with others studies, ${ }^{8-10}$ that postulated that chemical agents were efficient in reducing bad breath.

A few studies suggest the existence of oral malodor effects of flavoring agents present in oral care products. Morris, Read ${ }^{24}$ (1949) reported that dentifrices did present an effect of reducing oral malodor, while Brunette et al. ${ }^{9}$ (1998) suggested that the presence of flavoring agents could mask the presence of VSCs. Other authors ${ }^{11,12}$ suggested that flavor might act by altering the oral cavity clearance by inducing a fast and passing increase in the salivary flow immediately after the use of a dentifrice. In fact, this increase could accelerate and eliminate the bacteria by deglutition, thus reducing bad breath. It would be interesting to evaluate other flavor-containing 
dentifrices, in combination or in isolation, always respecting the complexity of the formulations and their effect associated with tooth brushing. However, further studies are necessary to explain the activity patterns of flavored dentifrices or its influence on morning breath.

\section{References}

1. Lee CH, Kho HS, Chung SC, Lee SW, Kim YK. The relationship between volatile sulfur compounds and major halitosisinducing factors. J Periodontol. 2003;74(1):32-7.

2. Goldberg S, Kozlovsky D, Gordon D, Gelernter I, Sintov A, Rosenberg M. Cadaverine as a putative component of oral malodor. J Dent Res. 1994;73(6):1168-72.

3. Persson S, Edlund MB, Claesson R, Carlsson J. The formation of hydrogen sulphide and methyl mercaptan by oral bacteria. Oral Microbiol Immunol. 1990;5(4):195-201.

4. Sharma NC, Galustians HJ, Qaqish J. The clinical effectiveness of a dentifrice containing triclosan and a copolymer for controlling breath odor measured organoleptically twelve hours after toothbrushing. J Clin Dent. 1999;10(4):131-4.

5. Faveri M, Hayacibara MF, Pupio GC, Cury JA, Tsuzuki CO, Hayacibara RM. A cross-over study on the effect of various therapeutic approaches to morning breath odor. J Clin Periodontol. 2006;33(8):555-60.

6. Rosenberg M, McCulloch CAG. Measurements of oral malodor: current methods and future prospects. J Periodontol. 1992;63(9):776-82.

7. Nogueira-Filho GR, Duarte PM, Toledo S, Tabchoury CPM, Cury JA. Effect of triclosan dentifrices on mouth volatile sulphur compounds and dental plaque trypsin-like activity during experimental gingivitis development. J Clin Periodontol. 2002;29(12):1059-64.

8. Niles HP, Miller S, Gaffar A. Mouth odor reduction by a sodium bicarbonate dentifrice. J Dent Res. 1993;72(4):24952.

9. Brunette DM, Proskin HM, Nelson BJ. The effects of dentifrice systems on oral malodor. J Clin Dent. 1998;9(3):7682.

10. Gerlach RW, Hyde JD, Poore CL, Stevens DP, Witt JJ. Breath effects of three marketed dentifrices: a comparative study evaluating single and cumulative use. J Clin Dent. 1998;9(4):83-8.

11. Bowen WH. The significance of toothpaste in oral hygiene. In: Embery G, Rölla G. Clinical and Biological Aspects of Dentifrices. Oxford University Press: New York; 1992. p. 916.

12. Davis WB. The function of toothpaste components. In: Jorgensen KD, editor. The Windsor Seminar on toothpaste stan-

\section{Conclusion}

Within the limitations of this study, it was concluded that the use of flavor-containing dentifrices seems to exert some action on morning bad breath in PHS, by decreasing VSCs levels, regardless of the amount of tongue coating.

dards with special emphasis on abrasion. Council of Public Health, Danish Dental Association; 1981.

13. Ainamo J, Bay I. Problems and proposals for recording gingivitis and plaque. Int Dent J. 1975;25(4):229-35.

14. Kozlovsky A, Gordon D, Gelernter I, Loesche WJ, Rosenberg M. Correlation between the BANA test and oral malodor parameters. J Dent Res. 1994;73(5):1036-42.

15. Rosenberg M, Kulkarni GV, Bosy A, McCulloch CA. Reproducibility and sensitivity of oral malodor measurements with a portable sulphide monitor. J Dent Res. 1991;70(11):143640.

16. Loesche WJ, Bretz WA, Lopatin D, Stoll J, Rau CF, Hillenburg KL et al. Multi-center clinical evaluation of a chairside method for detecting certain periodontopathic bacteria in periodontal disease. J Periodontol. 1990;61(3):189-96.

17. Loesche WJ, Kazor C. Microbiology and treatment of halitosis. Periodontol 2000. 2002:28(1):256-79.

18. Ayres M, Ayres M Jr, Ayres DL, Dos Santos AS. BioEstat 2.0: Aplicações estatísticas nas áreas das Ciências Biológicas e Médicas. Belém: Sociedade Civil Mamirauá, Brasília: CNPq; 2000.

19. Pedrazzi V, Sato S, Mattos MGC, Lara EHG, Panzeri H. Tongue-cleaning methods: a comparative clinical trial employing a toothbrush and a tongue scraper. J Periodontol. 2004;75(7):1009-12.

20. Bosy A, Kulkarni GV, Rosenberg M, McCulloch CA. Relationship of oral malodor to periodontitis: evidence of independence in discrete subpopulations. J Periodontol. 1994;65(1):37-46.

21. De Boever EH, Loesche WJ. Assessing contribution of anaerobic microflora of the tongue to oral malodor. J Am Dent Assoc. 1995;126(10):1384-93.

22. Miyazaki H, Sakao S, Katoh Y, Takehara T. Correlation between volatile sulfur-containing compounds and certain oral health measurements in the general population. J Periodontol. 1995;66(8):679-84.

23. Yaegaki K, Sanada K. Volatile sulfur compounds in mouth air from clinically healthy subjects and patients with periodontal disease. J Periodontal Res. 1992;27(4 Pt 1):233-8.

24. Morris PP, Read RR. Halitosis: variations in mouth and total breath odor intensity resulting from prophylaxis. J Dent Res. 1949;28(3):324-33. 